\title{
Effects of palatal augmentation prosthesis in oropharyngeal dysphagia in Chilean patients with tongue cancer: Cases report
}

\author{
Ana María Contreras, ${ }^{1}$ Alejandra Martínez, ${ }^{2}$ Ricardo Alarcón, ${ }^{3}$ \\ Vinka Devcic, ${ }^{4}$ Alfonso Catalán ${ }^{5^{*}}$
}

1. Graduate Student, Oral Prosthetic Rehabilitation Program, Department of Restorative, School of Dentistry, Universidad de Concepción, Concepción, Chile. 2. Professor, Oral Prosthetic Rehabilitation Program, Department of Restorative Dentistry, School of Dentistry, Universidad de Concepción, Chile. 3. Otolaryngologist, Service of Otolaryngology Guillermo Grant Benavente Hospital, Concepción, Chile. 4. Speech Language Pathologist, Service of Otolaryngology Guillermo Grant Benavente Hospital, Concepción, Chile.

5. Professor, Removable Prosthetics, Director of the Oral Prosthetic Rehabilitation Program, Department of Restorative Dentistry, School of Dentistry, Universidad de Concepción, Chile.

${ }^{*}$ Correspondence to: Dr. Alfonso Catalán | School of Dentistry, Universidad de Concepción | Casilla 160-C, Concepción, Chile | Phone number: 56-41-2204481 | Fax: 56-41-220 7440 |E-mail: acatalan@udec.cl Trabajo recibido el 05/07/2016. Aprobado para su publicación el 11/12/2016

\section{ABSTRACT}

Therapy of malignant neoplasms of the tongue or oropharynx may cause dysphagia Dysphagia in patients has negative significant social and psychological consequences. The six cases reports describe the effect of palatal augmentation prosthesis (PAP) on the management of dysphagia in patients with oral-pharyngeal cancer and as consequence of its treatment. The study also assesses PAP's effects on the quality of life of such patients.

KEYWORDS

Dysphagia, Tongue cancer, Palatal augmentation prosthesis, Quality of life.

Rev. Clin. Periodoncia Implantol. Rehabil. Oral Vol. 10(3); 156-158, 2017.

\section{INTRODUCTION}

Dysphagia is a common occurrence in patients with tongue cance (TC), and its treatment has been demonstrated to have significant influence on the function of the tongue during swallowing. ${ }^{(1-6)}$ In addition, there are consequences on the physical and mental condition of patients and it reduces their quality of life (QoL). ${ }^{(7,8)}$

Patients with oral and oropharyngeal cancer are usually treated with surgery, radiotherapy (RT), and chemotherapy (ChT), administered alone or in combination. ${ }^{(2-6,9)}$

The main post-radiotherapy sequelae are mucositis, xerostomia, dysgeusia, hoarseness, fibrosis and osteonecrosis.. ${ }^{(1,2)}$ Difficulties with speech, mastication and swallowing are also produced. ${ }^{(1,3-6)}$ Chemotherapeutic agents for head and neck cancer (HNC) can impact the ability of patients to swallow thus causing nutritional problems. (3)

The incidence of post-treatment dysphagia in patients with HNC has previously been reported to be between $50 \%$ and $60 \%$. ${ }^{(7)}$ Patients who exhibit worsened swallow function have longer oral transit times, significantly increased pharyngeal transit times, oral and pharyngea residues, posterior spillover, and shorter duration of cricopharyngea opening. ${ }^{(4)}$ Dysphagia produces a series of consequences such as penetration, aspiration, regurgitation, pneumonia, dehydration, malnutrition, weight loss, and negative impact on QoL. ${ }^{(3,8,10-13)}$ Furthermore, dysphagia has the potential of life-threatening infection due to aspiration pneumonia. $^{(1)}$

There is ample evidence of the use of palatal augmentation prosthesis (PAP) to enhance dysphagia and dysartria. ${ }^{(4,9,10,12,13)}$ PAP are used to mode and lower the palatal vault to offer new contact for the remaining portion of the resected tongue. Thus, the patients can performed the functions of swallowing and speech. ${ }^{(9,10,12)}$ Despite this evidence elsewhere, use of PAP to manage dysphagia post cancer treatment has not been reported in Chilean literature. Besides, this report describes a tecnique for PAP where palatal surface was reproduced by a functional impression of the tongue dorsal surface by means of tissue conditioner.

Studies on the QoL of patients with HNC have shown high levels of emotional anguish, physical limitations and damage in social relations. $(7,8,14,15)$ Thus, the aims of this cases report study was to describe the effect of PAP and to evaluate the effects on the quality of life of six Chilean patients with post-treatment dysphagia of tongue cancer.

\section{CASES REPORT METHODS}

This clinical report consisted of six patients with tongue cancer treated at the Department of Otolaryngology-Head and Neck Surgery, of the Guillermo Grant Benavente Hospital, at Concepción, Chile. All the patients were referred by their otolaryngologist (OTL) to the Oral Prosthetic Rehabilitation Program at the School of Dentistry at Universidad de Concepción for fabricated dental prosthesis. Patients were evaluated before and after the PAP placement by an OTL, a speech language pathologist (SLP), and a prosthodontist. This study was approved by the Ethics Committee of Universidad de Concepción, and Guillermo Grant Benavente Hospital. Informed consent was obtained from all subjects.

A clinical swallow assessment was conducted by the SLP. Motor evaluation of the oral structure and swallowing trials with three different food textures were performed.

A fiberoptic endoscopic evaluation of swallowing (FEES) procedure was performed by an OTL, using an (Olympus endoscope, model 1420721, Japan), with two different consistencies (liquid and paste). Solid foods were excluded because not all patients could ingest this consistency. Alterations were observed from the pharynx, such as detection of retention of food and fluids in the pharynx after the swallow, several swallows per bolus, penetration into the airway, and posterior spillover.

Clinical evaluation was done by a prosthodontist. Xerostomia was assessed by self-reported oral dryness and by clinically assessing dry mouth.

Conventional removable complete dentures (RCD) and removable partial dentures (RPD) with forged steel retainer were fabricated by the prosthodontist with maximum palatal coverage. The patient was given a trial period from two weeks to one month in order to adjust to these new prostheses. Then a functional impression of the dorsal surface of the tongue was made. A thick mix of tissue-conditioning material (CoeComfort, GC American Inc. Alsip IL, USA) was placed on the palatal external surface of the prosthesis, and then reshaped by the tonguepalate contact during swallowing water (Figure 1. A, B). Incremental addition of tissue conditioner was sustained until a swallowing reflex was produced, with the following characteristics: comfort swallowing, ability to swallow a sip of water once, and lack of cough reflex after swallowing. The new contours of palatal vaults were processed with transparent heatcuring acrylic resin (Meliodent, Heraeus, Gruner Weg, Germany). 
To assess the QoL of patients, the Spanish validation in Chilean patients of the University of Washington Head and Neck Cancer Quality of Life Questionnaire (UW-QoL) version 4, was applied. After two to five months of PAP placement, clinical evaluation of swallowing, FEES and Questionnaire UW-QoL were reapplied.

\section{PARTICIPANS}

Patient A: This was a 61-year-old man who had been diagnosed with squamous cell carcinoma (SCC) of the base of the tongue (T3N2cMO). Surgery with bilateral neck dissection was performed, followed by RT, and ChT. Xerostomia, trismus, dysphonia and osteomyelitis were post treatment complications. Intraoral examination revealed a partially edentulous non-denture wearer. In the swallowing evaluation, a mild degree of oropharyngeal dysphagia (OPD) was diagnosed, characterized by inability for solid food intake, movement of neck when swallowing, nasal regurgitation, escape of food out the mouth, increased oral transit times, presence of cough, as well as liquid and paste oral and pharyngeal residues. Posterior spillover with liquid was also observed. RPD-type PAP and RPD were fabricated. After five months wearing dentures and receiving SLP-therapy, numerous improvements were observed in swallowing, which included the ability to eat solid food, decreased ora transit time, disappearance of escape foods out of mouth, no cough, no nasal regurgitation, no oral and pharyngeal residues, no posterior spillover after swallowing and no movement of neck swallowing. The total index of QoL had increased from 76 to 81.
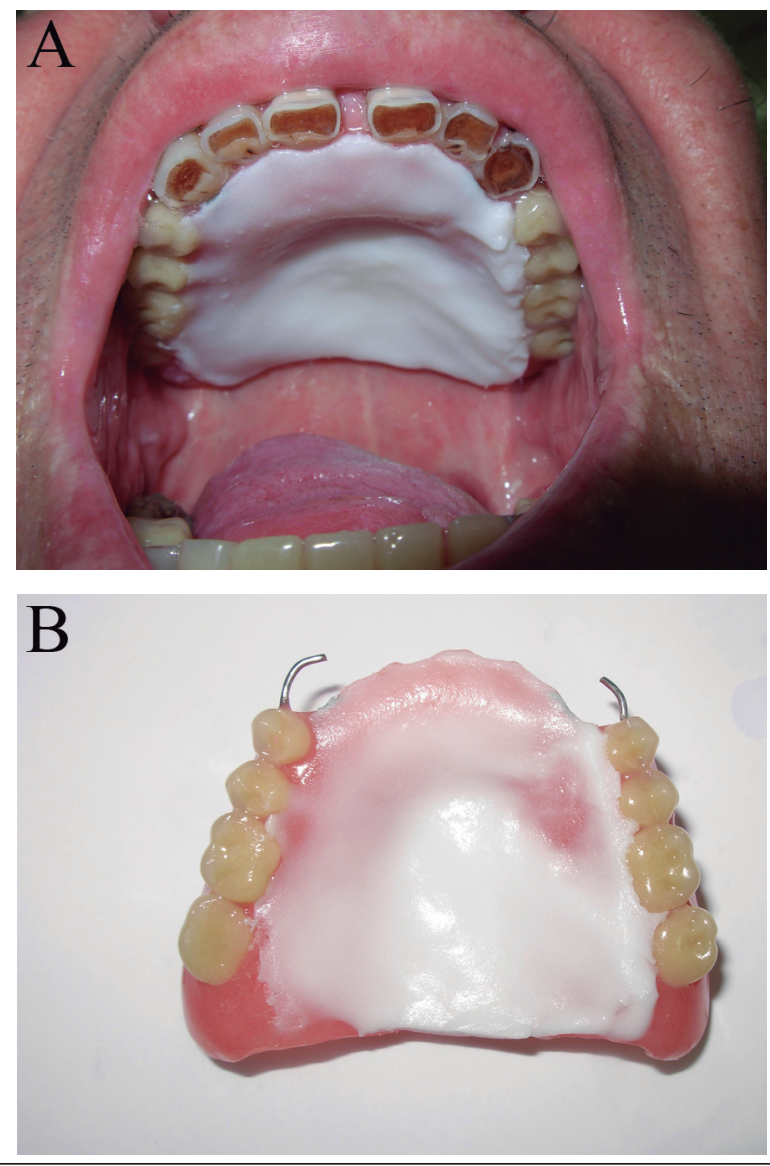

Figure 1. (A) Tissue conditioner impression material applied on the maxillary denture palate, so tongue can mold material during speech and swallowing. (B) Maxillary occlusal view of incremental addition of tissue conditioner.

Patient B: This was a 58-year-old male who was treated with RT and ChT for SCC of the base of tongue and pharynx (T2NOMO). Xerostomia and tinitus were post treatment alterations. Clinical examination showed that the patient wearing maxillary and mandibular RCD no longer provided confortable function. Clinical swallow assessment and FEES revealed a mild degree of OPD branded by inability for solid food intake, presence of cough during eating, paste oral and pharyngeal residues, and posterior spillover of liquids and paste. The patient received a RCD maxillary-type
PAP and a mandibular RCD. After four months wearing prostheses and without complementary therapy with SLP, the patient showed a greater improvement in his ability to eat solid food; there was no cough, no oral residues, and no posterior spillover with both consistencies. UW-QoL composite score with PAP was significantly improved from 74 to 85 after PAP was placed. The patient reported improvements in mastication, aesthetics, ability to socialize and comfort levels during the observed time period.

Patient C: This was a 68-year-old man who had received RT and ChT treatment for SCC on the posterior lateral border and on the ventral of the tongue with compromise of the oral floor (T4N2aM0). As a result of cancer treatment, xerostomia, trismus, tinitus and dysphonia were found. Oral examination showed a completely edentulous patient, wearing denture with no stability. Swallowing evaluation revealed a mild degree of OPD with inability for solid food intake, cough, pharyngeal residue after swallowing with paste, and posterior spillover with liquids and paste. The prosthetic treatment was RCD maxillary type-PAP and mandibular RCD. Four months later, after wearing prostheses and receiving therapy with SLP, many improvements were observed in his swallowing, which included the ability to eat solid food, vanishing cough, disappearance of pharyngeal residues and posterior spillover after swallowing. Despite these improvements, oral residues after swallowing appeared. The patient was pleased with his PAP and expressed improved satisfaction. His UW-QoL composite score increased from 56 to 74 .

Patient D: This was a 68-year-old man who presented SCC of the base of the tongue, left tonsil, and pharynx. Cervical node metastatic was detected (T3N2aM0). The patient underwent surgery with bilateral neck dissection, RT, and ChT. The intraoral examination exhibited a partially edentulous non-denture wearer. Post treatment complications included xerostomia, ankyloglossia and dysarthria. Clinical swallow assessment diagnosed a mild degree of OPD characterized by inability for solid food intake, cough, increased oral transit time, paste oral and pharyngeal residues, posterior spillover and penetration with liquids and paste. Maxillary RPD-type PAP and mandibular RPD were fabricated. The patient did not wear the prostheses permanently and declined therapy with SLP. Three months after insertion of prostheses, the patient showed only one improvement: solid food intake. UW-QoL composite score experienced a slight decrease from 73 to 69 after wearing PAP.

Patient E: This was a 78-year-old man with a history of SCC of the ventral surface and lateral posterior tongue border (T2NOM0), treated by surgery with bilateral node neck dissection, RT, and ChT. Oral examination revealed an edentulous patient with xerostomia. He was wearing only maxillary RCD and was unable to wear his mandibular complete denture. The swallowing evaluation revealed a mild degree of OPD featuring inability for solid food intake, escape of food out the mouth, increased oral transit time, movement of neck, cough, as well as liquids and paste oral and pharyngeal residues, and posterior spillover with liquid.

Prosthetic rehabilitation included RCD maxillary denture-type PAP and a mandibular RCD. The patient did not wear the PAP permanently during the adaptation period. After two months of intermittent wear and without complementary therapy with SLP, various improvements were found including ability to eat solid food, disappearance of escape foods out of mouth, disappearance of cough and of movement of neck. Also, oral and pharyngeal residues with liquids vanished. With PAP the UWQoL composite score experienced improvements from 54 to 65.

Patient F: This was a 50-year-old man who was treated for SCC on the right lateral border of the tongue and the oral floor (T4NOMx). His medical history revealed that the patient had received surgery with bilateral node neck dissection and RT. The patient presented xerostomia, dysartria and depression as adverse effects of cancer treatment. Intraoral examination exposed a partially edentulous non-denture wearer patient. Clinical evaluation of swallowing and FEES were performed and a diagnosis of OPD was made. OPD was typified by restrictive solid food intake, increased oral transit time and oral and pharyngeal residues with paste, posterior spillover and penetration of liquids and paste. Prosthetic treatment consisted of RPD maxillary-type PAP. The mandibular removable prosthesis was excluded from the treatment options because of severe atrophy of ridge. After three months wearing PAP in an intermittent way and without therapy with SLP, only one improvement was observed in his dysphagia, which consisted in the disappearance of oral residues after swallowing and liquid penetration. In addition, a significant improvement was observed in speech, which allowed him to move away from the depression he had developed after surgery. The results of the survey UW-QoL composite score experienced a great improvement, shifting from 31 to 65 post insertion of PAP.

\section{DISCUSSION}


Dysphagia is an important symptom of tongue cancer in latter stages. It also represents a significant complication in cancer therapy. ${ }^{(5,6,13)}$ Negative effects that surgery, ChT, RT, and multimodality treatment protocols have on tongue function have been documented. ${ }^{(2-6,9,12)}$ The results of the current cases report show that PAP was effective as a complement therapy of dysphagia in post-tongue cancer patients. PAP positively affected oral and pharyngeal phases of swallowing in all patients. These findings are in agreement with results from previous studies. $(9,10,13)$

PAP fabrication commonly uses a technique to reproduce palatal vault by means of different impression materials, except for tissue conditioners. $(9,10)$ This report describes an innovative technique for PAP fabrication where functional impression of the dorsal surface of the tongue was made precisely with tissue conditioner, reshaped by tongue-palate contact during swallowing water.

One disabling complication was inadequate bolus propulsion, leaving ora or pharyngeal residue after swallowing. Fluid intake improved after insertion of PAP as intraoral liquid residues disappeared in all patients, and pharyngeal residues in $67 \%$ (two out of three) of patients. Swallowing paste was also affected favorably with PAP. For $60 \%$ (three out of five) of patients these ora level residues disappeared, while for $33 \%$ (two out of six) pharyngeal level also disappeared. These data support findings in previous studies. ${ }^{(11,13)}$

Retention of pharyngeal residues increases the risk of penetration and aspiration; therefore, it should be treated promptly. ${ }^{(6,11)}$

From the patients who had multiple swallows with liquid, $75 \%$ (three of out four) improved swallowing by wearing PAP. Fifty percent (three out of six) decreased the number of swallows necessary to clean a bolus of paste but no patient was able to swallow in one go. Others researchers have also found that higher viscosity of the bolus is more difficult to swallow among this kind of patients. ${ }^{(4,11)}$

The posterior spillover of food from the oral cavity into the pharynx is a sign presented in patients treated from HNC. This is due to insufficient mobility of the base of the tongue to rise and reach the soft palate, which prevents them from achieving the correct closure of the posterior oral sphincter. ${ }^{(12)}$ All patients in this study showed posterior spillover of liquids, $50 \%$ (three out of six) improved after the insertion of PAP. Fifty percent of patients (two out of four) with posterior spillover with paste improved by wearing PAP.

Dysphagia in post-tongue cancer patients can cause a decrease in daily living activities and QoL..(7,9,15) In the current study, the global QoL score before prosthodontic treatment was $60.6( \pm 17.5)$ on average, and the most affected domains were swallowing $(100 \%)$ and saliva (xerostomia; $100 \%)$. After prosthodontic treatment with PAP, the global QoL score was $73.0( \pm 8.5)$ on average. This means that prosthetic therapy with PAP had a positive impact on the QoL of patients in the present study.

This clinical report demonstrates effective collaboration between dental, OTL and SPL professionals for patients who have suffered significant functional disabilities due to tongue cancer and its treatment sequelae. The ability of clinicians to rehabilitate dysphagia is essential for the future of the QoL for patients treated from tongue cancer.

\section{CONCLUSION}

In this limited sample size cases report, results indicate that after the insertion of PAP, with palatal surface reproduced by a functional impression made of tissue conditioner, patients' Quality of Life increased and dysphagia due to tongue cancer and its treatment improved.

\section{ACKNOWLEDGEMENTS}

We thank all patients for their perseverance to attend to all their controls.

\section{References}

1. Shune SE, Karnell LH, Karnell MP, Van Daele DJ, Funk GF. Association between severity of dysphagia and survival in patients with head and neck cancer. Head Neck. 2012;34:776-784

2. Platteaux N, Dirix P, Dejaeger E, Nuyts S. Dysphagia in head and neck cance patients treated with chemoradiotherapy. Dysphagia. 2010;25:139-152.

3. Barringer DA, Hutcheson KA, Sturgis EM, Kies MS, Lewin JS. Effect of induction chemotherapy on speech and swallowing function in patients with oral tongue cancer. Head Neck. 2009;31:611-617.

4. Pauloski B. Rehabilitation of dysphagia following head and neck cancer. Phys Med Rehabil Clin N Am. 2008;19: 889-928.

5. Nguyen NP, Moltz CC, Frank C, Vos P, Smith HJ, Nguyen PD, et al. Impact of swallowing therapy on aspiration rate following treatment for locally advanced head and neck cancer. Oral Oncol. 2007;43:352-7.

6. Gaziano JE. Evaluation and managment of oropharyngeal dysphagia in head and neck cancer. Cancer Control. 2002;9:400-408.

7. Nguyen NP, Frank C, Moltz CC, Vos P, Smith HJ, Karlsson U, et al. Impact of dysphagia on quality of life after treatment of head-and-neck cancer. Int J Radiat Onco Biol Phys. 2005;61:772-778

8. Chen A, Frankowski R, Bishop-Leone J, Hebert T, Leyk S, Lewin J et al. The development and validation of a dysphagia-specific quality of life questionnaire for patients with head and neck cancer. Arch Otolaryngol Head and Neck Surg. 2001;127: 870-876.
9. Okayama H, Tamura F, Kikutani T, Kayanaka H, Katagiri $\mathrm{H}$, Nishiwaki K. Effects of a palatal augmentation prosthesis on lingual function in postoperative patients with oral cancer: coronal section analysis by ultrasonography. Odontology. 2008;96:26-31.

10. Watanabe S, Oh-Shige H, Miyachi H, Gotoh M, Ariji E, Shimozato K. Use of palatal augmentation prostheses to maintain speech and swallowing function in patients with amyotrophic lateral sclerosis. J Oral Maxillofac Surg Med Pathol. 2012; 24:119-123.

11. Yeates E, Molfenter S, Steele C. Improvements in tongue strength and pressuregeneration precision following a tongue-pressure training protocol in older individuals with dysphagia: Three case reports. Clin Interv Aging. 2008;3:735-747.

12. Murry T, Carrau R. Clinical management of swallowing disorders. $2^{\mathrm{a}}$ ed. San Diego: Plural Publishing; 2006. p.3-5, 10, 156-161, 170-183.

13. Marunick M, Tselios N. The efficacy of palatal augmentation prostheses for speech and swallowing in patients undergoing glossectomy: A review of the literature. J Prosthet Dent. 2004;91:67-74.

14. Nazar G, Garmendia M, Royer M, McDowell J, Weymuller E, Yueh B. Spanish validation of the University of Washington Quality of Life questionnaire for head and neck cancer patients. Otolaryngol Head Neck Surg. 2010;143:801-807.

15. Roger SN, Gwanne S, Lowe D, Humphris G, Yueh B, Weymuller EA Jr. The addition of mood and anxiety domains to the University of Washington Quality of Life scale. Head Neck. 2002;24:521-529. 